\title{
Indoor visible light communications: performance evaluation and optimization
}

\author{
Sheng-Hong Lin ${ }^{1,2}$, Cheng Liu ${ }^{3}, \mathrm{Xu} \mathrm{BaO}^{1}$ and Jin-Yuan Wang ${ }^{1,2^{*}}$ (1)
}

\begin{abstract}
For indoor visible light communication (VLC), much work has been done when the noise is independent of the input signal. However, less effort is made when the VLC system suffers from the input-dependent noise. Considering the input-dependent noise, this paper focuses on the performance analysis and optimization for indoor VLC system. The Lambertian emission-based channel model and on-off keying modulation are employed. According to the system model, the bit error rate (BER) with a closed-form expression is derived. To enhance the system performance, an optimization problem that minimizes the BER by tilting the receiver plane is formulated. By solving the problem, the optimal tilting angle of the receiver is obtained. Simulation results verify the derived expression of BER. It is also shown that the BER is strongly affected by the input-dependent noise. Moreover, the optimal tilting angles for the receiver at any position are obtained, which can provide some insights for practical system design.
\end{abstract}

Keywords: Visible light communications, BER, Input-dependent noise, Tilting receiver plane

\section{Introduction}

In the past 10 years, the visible light communications (VLC) has gained substantial attention [1]. In VLC, the light-emitting diodes (LEDs) are often employed as the transmitter, while the photodiodes (PDs) are used as the receiver. The illumination and communication in VLC can be implemented at the same time. Moreover, VLC has many advantages, e.g., unlicensed spectrum, immunity to radio frequency interference, and high transmission rate. Consequently, VLC has become one of the most promising candidates for indoor wireless access in the forthcoming fifth generation (5G) communications [2].

The VLC can be implemented in both outdoor and indoor scenarios. For the outdoor scenario, the VLC is mainly used for the intelligent transportation system $[3,4]$. However, much researchers concentrate on the indoor VLC. For the indoor scenario, a fundamental analysis for VLC is provided in [5]. In [6], the channel capacity bounds of VLC are derived by considering the

\footnotetext{
*Correspondence: jywang@njupt.edu.cn

${ }^{1}$ Jiangsu Key Laboratory of Traffic and Transportation Security, Huaiyin Institute of Technology, 223003 Huaian, China

${ }^{2}$ Key Lab of Broadband Wireless Communication and Sensor Network

Technology, Nanjing University of Posts and Telecommunications, 210003 Nanjing, China

Full list of author information is available at the end of the article
}

constraints of the input signal. Moreover, ref. [7] derives a much tighter upper bound on the channel capacity for indoor VLC. By using pulse amplitude modulation and the inverse source coding, the capacity of VLC is further analyzed in [8]. In [9] and [10], the asymmetrically clipped optical-orthogonal frequency division multiplexing (ACO-OFDM) and the direct current optical-OFDM (DCO-OFDM) are discussed for VLC, respectively. By employing the color shift keying (CSK) and the generalized space shift keying (GSSK), the error performance of VLC is analyzed in [11] and [12], respectively. In [13] and [14], an optimal spread code and an adaptive equalizer for multi-path dispersion in VLC are proposed. It should be emphasized that the noise and the input signal in [5]-[14] are assumed to be independent of each other. However, owing to the stochastic behavior of photon emission in LED, the noise in VLC depends on the input signal [15]. Note that the channel capacity of VLC with the input-dependent noise is investigated in [16], and the mutual information for optical spatial modulation with the input-dependent noise is discussed in [17]. However, the link reliability of the VLC (such as bit error rate (BER)) with the input-dependent noise has not been studied.

In previous work, the normal vectors of the transceiver planes are often supposed to be perpendicular to the 
ceiling [18-20]. By using this setup, the performance analysis for VLC is more convenient. However, the system performance also degrades dramatically when the distance between the transceiver is large [21]. In other words, when the transceiver distance becomes large, the received signal-to-noise ratio (SNR) at the $\mathrm{PD}$ will be very small, and thus the BER will be very large. When the distance of the transceiver is large, how to improve the system performance becomes an important problem to be solved. As we know, if the PD inclines its orientation toward the LED, the received SNR will be enhanced observably. Therefore, to obtain better system performance, the optimal tilting angle of the PD should also be investigated.

\section{Methods}

In this paper, an indoor VLC system with a single transmitter and a single receiver is considered. The Lambertian emission-based channel model and the on-off keying (OOK) are employed. In the system, the noises include two parts: input-dependent noise and inputindependent noise. In the presence of two kinds of noises, the BER of the system is analyzed. As a special case, the BER with only the input-independent noise is also shown. By minimizing the BER, an optimization problem is formulated to improve the system performance. Then, the optimization problem is solved by using the principle of the convex optimization. After solving the problem, the optimal tilting angle of the receiver is obtained. The derived theoretical results of BER are all confirmed by using the Monte-Carlo simulations. Moreover, the proposed performance enhancement scheme will expedite the analysis and help gain deeper insights for VLC.

\section{System model}

Consider a classic indoor VLC system with a single LED and a single PD, as depicted in Fig. 1 . The room size is set to be $J \times K \times L$. The LED is employed as the transmitter, which is fixed on the center of the ceiling. The position of the LED is supposed to be $[u, v, w]$. The PD as the receiver is deployed on a horizontal plane, whose position is assumed to be $[a, b, c]$. The PD can move to any place of the receiver plane and can change its direction by tilting a proper angle.

\subsection{Transmitter}

At the transmitter, the emitted instantaneous optical intensity signal by the LED is denoted as $X$, and the average optical intensity of the LED is denoted as $P$. Without loss of generality, an intensity modulation scheme, OOK modulation, is employed. We assume that $X$ is equal probably generated from the OOK modulation constellation, and thus $X$ belongs to the following set

$$
X \in\{0,2 P\}
$$

\subsection{Channel model}

For indoor VLC, the channel gain can be modeled by the Lambertian emission [22]

$$
h=\frac{(m+1) A}{2 \pi d^{2}} \cos ^{m}(\alpha) \cos (\beta)
$$

where $m=-\ln 2 / \ln \left(\cos \Phi_{1 / 2}\right)$ is order of the Lambertian emission, and $\Phi_{1 / 2}$ is the semi-angle at half power of the LED. $A$ is the physical area of the receiver. $d$ is the transceiver distance. $\alpha$ is the irradiance angle of the LED, while $\beta$ is the incidence angle of the PD. Note that both the optical filter gain and the concentrator gain of the PD are

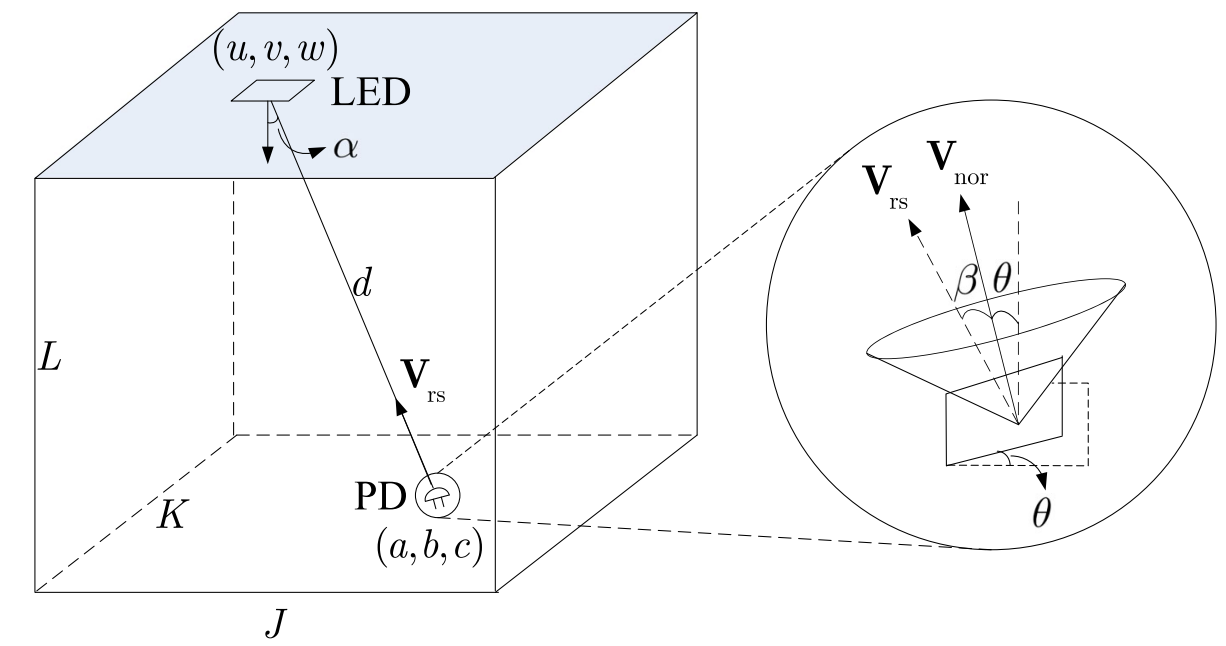

Fig. 1 A classic point-to-point indoor VLC system 
set to be one, and thus they are omitted in (2). Moreover, $h$ in VLC is non-negative and real, and thus $\alpha, \beta \in[0, \pi / 2]$.

According to Fig. 1, the geometrical relationship of the transceiver is given by

$$
\cos (\alpha)=\frac{w-c}{d}
$$

Define $\mathbf{V}_{\mathrm{rs}}$ as the vector pointing from the receiver to the transmitter, and $\mathbf{V}_{\text {nor }}$ as the unit normal vector of the receiver plane. Consequently, we have

$$
\cos (\beta)=\frac{\left\langle\mathbf{V}_{\text {nor }}, \mathbf{V}_{\mathrm{rs}}\right\rangle}{\left\|\mathbf{V}_{\text {nor }}\right\| \cdot\left\|\mathbf{V}_{\mathrm{rs}}\right\|}
$$

where $\langle\cdot, \cdot\rangle$ represents the inner product operator, and $\|\cdot\|$ represents the norm operator.

In this paper, assume that the position of the PD is not changed by tilting the receiver plane. Therefore, $\mathbf{V}_{\mathrm{rs}}=$ $[u-a, v-b, w-c], \mathbf{V}_{\text {nor }}=[\cos \varphi \sin \theta, \sin \varphi \sin \theta, \cos \theta]$, where $\theta$ and $\varphi$ are the tilting angle of the PD and the azimuth angle. The tilting angle and the azimuth angle are shown in Figs. 1 and 2, respectively. Assume that the projection of the LED onto the XY plane is located in the $i$-th quadrant, and $\varphi$ can be expressed as [21]

(c)
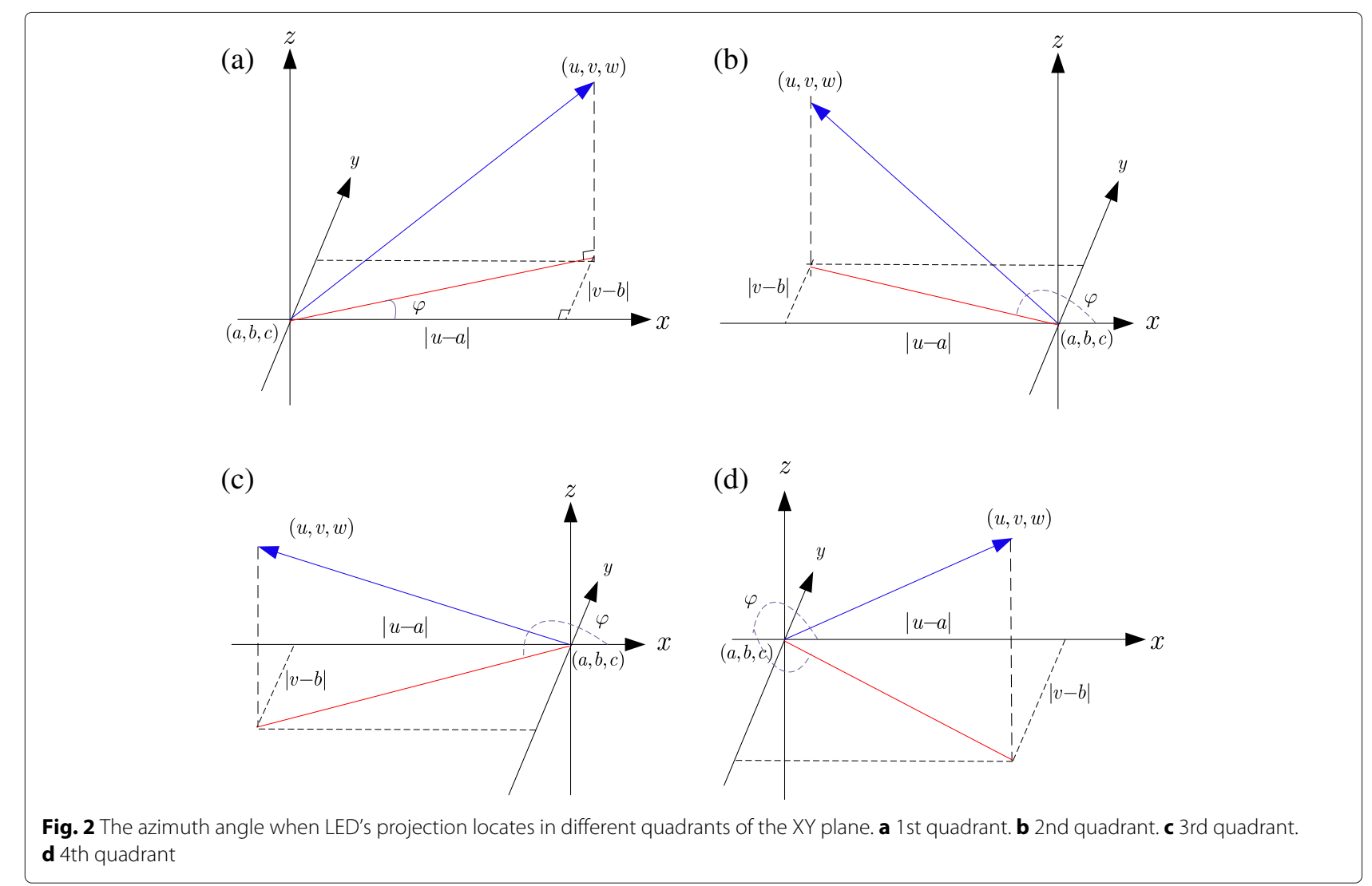

$$
\varphi= \begin{cases}\arctan \left|\frac{v-b}{u-a}\right|, & i=1 \\ \pi-\arctan \left|\frac{v-b}{u-a}\right|, & i=2 \\ \pi+\arctan \left|\frac{v-b}{u-a}\right|, & i=3 \\ 2 \pi-\arctan \left|\frac{v-b}{u-a}\right|, & i=4\end{cases}
$$

Furthermore, (4) can be written as

$$
\begin{aligned}
\cos (\beta)= & {[(u-a) \cos \varphi \sin \theta+(v-b) \sin \varphi \sin \theta} \\
& +(w-c) \cos \theta] / d
\end{aligned}
$$

Substituting (3) and (6) into (2), the channel gain can be further expressed as

$$
\begin{aligned}
h= & \frac{(m+1) A(w-c)^{m}}{2 \pi d^{m+3}}[(u-a) \cos \varphi \sin \theta \\
& +(v-b) \sin \varphi \sin \theta+(w-c) \cos \theta]
\end{aligned}
$$

where $d=\sqrt{(u-a)^{2}+(v-b)^{2}+(w-c)^{2}}$. As can be seen in (7), when the positions of the LED and PD are fixed, the parameters $a, b, c, u, v$ and $w$ are constants. Moreover, according to (5), the azimuth angle $\varphi$ is also a constant. This indicates that given the positions of the LED and PD, the channel gain $h$ is a function of the tilting angle $\theta$. 


\subsection{Receiver}

At the receiver, the corrupted noises include thermal noise, amplifier noise, and shot noise. All of them can be modeled by Gaussian distributions [23]. However, the first two noises are independent of the input signal, while the shot noise depends on the input signal $[16,17]$. So, the received electrical signal $Y$ at the $\mathrm{PD}$ is given by

$$
Y=\operatorname{rh} X+\sqrt{\operatorname{rh} X} Z_{1}+Z_{0}
$$

where $r$ is the optoelectronic conversion factor of the PD. $Z_{0} \sim\left(0, \sigma^{2}\right)$ and $Z_{1} \sim\left(0, \varsigma^{2} \sigma^{2}\right)$ are the input-independent noise, and the input-dependent noise, respectively. $\varsigma^{2} \geq 0$ is the scaling factor.

\section{BER analysis}

In wireless communications, BER is one of the commonly used indicators to reflect the system performance. Here, the BER and the optimal detection threshold for the considered VLC system will be investigated. Moreover, the behavior of the derived expressions will also be analyzed.

\subsection{Derivations of BER and optimal detection threshold}

From (7), it can be seen that $h$ is fixed when the coordinates of LED and PD are pre-given. When OOK is employed, the BER for VLC can be expressed as [24]

$$
\text { BER }=\operatorname{Pr}(\text { off }) \operatorname{Pr}(\text { on } \mid \text { off })+\operatorname{Pr}(\text { on }) \operatorname{Pr}(\text { off } \mid \text { on })
$$

where $\operatorname{Pr}($ off $)=\operatorname{Pr}($ on $)=1 / 2$. In the following, the conditional error probabilities $\operatorname{Pr}$ (on $\mid$ off) and $\operatorname{Pr}($ off $\mid$ on) will be analyzed, respectively.
According to (8), conditioned on $X, Y$ is a Gaussian distribution. Therefore, the conditional probability density function (PDF) $f_{Y \mid X}(y \mid x)$ is obtained as

$f_{Y \mid X}(y \mid x)=\frac{1}{\sqrt{2 \pi\left(1+r h x \varsigma^{2}\right)} \sigma} \exp \left[-\frac{(y-r h x)^{2}}{2\left(1+r h x \varsigma^{2}\right) \sigma^{2}}\right]$

In this case, the detection threshold at the receiver is assumed to be $\eta$, which can be an arbitrary real number. Let $\chi=2 r h P$, and then $\operatorname{Pr}$ (off $\mid$ on $)$ can be derived as

$$
\begin{aligned}
\operatorname{Pr}(\text { off } \mid \text { on }) & =\int_{-\infty}^{\eta} \frac{e^{-\frac{(y-\chi)^{2}}{2\left(1+\chi \varsigma^{2}\right) \sigma^{2}}}}{\sqrt{2 \pi\left(1+\chi \varsigma^{2}\right)} \sigma} \mathrm{d} y \\
& =\mathcal{Q}\left(\frac{\chi-\eta}{\sqrt{1+\chi \varsigma^{2}} \sigma}\right)
\end{aligned}
$$

where $\mathcal{Q}(x)=\int_{x}^{\infty} e^{-t^{2} / 2} / \sqrt{2 \pi} \mathrm{d} t$.

Similarly, $\operatorname{Pr}$ (on $\mid$ off) can be derived as

$$
\begin{aligned}
\operatorname{Pr}(\text { on } \mid \text { off }) & =\int_{\eta}^{\infty} \frac{1}{\sqrt{2 \pi} \sigma} e^{-\frac{y^{2}}{2 \sigma^{2}}} \mathrm{~d} y \\
& =\mathcal{Q}\left(\frac{\eta}{\sigma}\right)
\end{aligned}
$$

Because $\operatorname{Pr}($ on $)=\operatorname{Pr}($ off $)=0.5$, (9) can be written as

$$
\operatorname{BER}_{\varsigma^{2}>0}=\frac{1}{2}\left[\mathcal{Q}\left(\frac{\chi-\eta}{\sqrt{1+\chi \varsigma^{2}} \sigma}\right)+\mathcal{Q}\left(\frac{\eta}{\sigma}\right)\right]
$$

In (13), the BER is a function of $\eta$. Figure 3 shows the value of BER versus $\eta$ with different $\varsigma$ when $r=0.9$, $(a, b, c)=(2 \mathrm{~m}, 2 \mathrm{~m}, 1.5 \mathrm{~m}),(u, v, w)=(2.5 \mathrm{~m}, 2.5 \mathrm{~m}, 3 \mathrm{~m})$, $P=55 \mathrm{dBm}$ and $\sigma=1$. It can be observed that for a

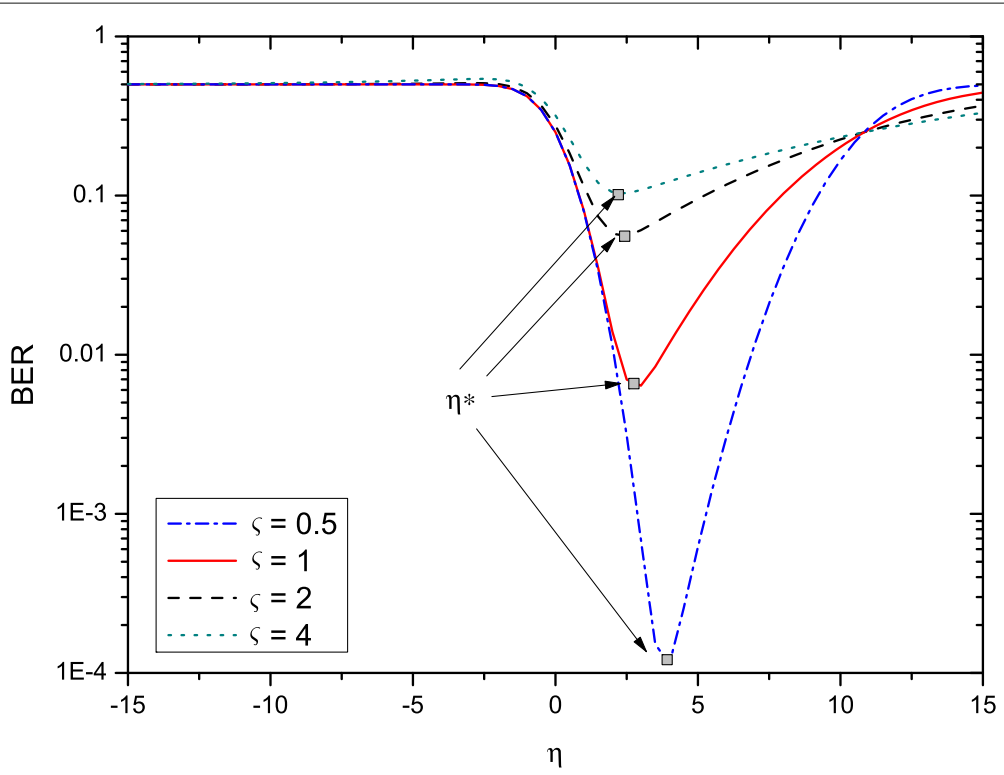

Fig. 3 The value of BER versus $\eta$ with different $\varsigma$ when $r=0.9,(a, b, c)=(2 \mathrm{~m}, 2 \mathrm{~m}, 1.5 \mathrm{~m}),(u, v, w)=(2.5 \mathrm{~m}, 2.5 \mathrm{~m}, 3 \mathrm{~m}), P=55 \mathrm{dBm}$ and $\sigma=1$ 
given $\varsigma$, the BER curve has a peak value and a valley value. As it is well known, the optimum detection threshold $\eta^{*}$ corresponds to the valley value of BER. By analyzing (13), the following theorem is obtained.

Theorem 1 For the system model in (8), the optimal detection threshold $\eta^{*}$ when $\varsigma>0$ is given by

$$
\eta^{*}=\frac{\sqrt{1+\chi \varsigma^{2}+\frac{\varsigma^{2} \sigma^{2}\left(1+\chi \varsigma^{2}\right) \ln \left(1+\chi \varsigma^{2}\right)}{\chi}}-1}{\varsigma^{2}}
$$

Proof See Appendix 1.

Substituting (14) into (13), the BER when $\varsigma^{2}>0$ can be finally written as

$$
\begin{aligned}
\operatorname{BER}_{\varsigma^{2}>0} & =\frac{1}{2}\left[\mathcal{Q}\left(\frac{\left.\sqrt{1+\chi \varsigma^{2}}-\sqrt{1+\frac{\varsigma^{2} \sigma^{2} \ln \left(1+\chi \varsigma^{2}\right)}{\chi}}\right)}{\varsigma^{2} \sigma}\right)\right. \\
& \left.+\mathcal{Q}\left(\frac{\sqrt{1+\chi \varsigma^{2}+\frac{\varsigma^{2} \sigma^{2}\left(1+\chi \varsigma^{2}\right) \ln \left(1+\chi \varsigma^{2}\right)}{\chi}}-1}{\varsigma^{2} \sigma}\right)\right]
\end{aligned}
$$

\subsection{Behavior analysis}

By analyzing Theorem 1, the changing trend of $\eta^{*}$ with 5 can be obtained in the following theorem.

Theorem 2 When $\chi$ is very small, the value of $\eta^{*}$ in (14) increases with the increase of 5 . However, when $\chi$ is very large, the value of $\eta^{*}$ in (14) decreases with the increase of 5 .

\section{Proof See Appendix 2.}

In Theorem 1, it can be seen that $\eta^{*}$ is a function of $\varsigma$. To show the relationship between $\eta^{*}$ and $\varsigma$, Fig. 4 shows the value of $\eta^{*}$ versus $\varsigma$ with different $P$ when $r=0.9$, $h=8.335 \times 10^{-7}$, and $\sigma=1$. As is observed, when $P$ is small, the value of $\eta^{*}$ increases with the increase of $\zeta$. However, when $P$ is large, the value of $\eta^{*}$ decreases with 5 . This result also verifies the correctness of Theorem 2.

By analyzing (15), the asymptotic behavior of the BER is derived in the following theorem.

Theorem 3 The asymptotic behavior of the BER in (15) is given by

$$
\lim _{\zeta \rightarrow 0} \mathrm{BER}_{\varsigma^{2}>0}=\mathrm{BER}_{\varsigma^{2}=0}=\mathcal{Q}\left(\frac{\chi}{2 \sigma}\right)
$$

\section{Proof See Appendix 3.}

It can be known from Theorem 3 that when $\varsigma \rightarrow 0$, the VLC system with input-dependent noise achieves the same BER performance as the VLC system with only input-independent noise (i.e., $\varsigma=0$ ). In this paper, Eq. (16) can be used as a benchmark.

\section{Problem formulation and solving}

In the above section, the BER has been analyzed for the indoor VLC. In this section, an optimization problem to

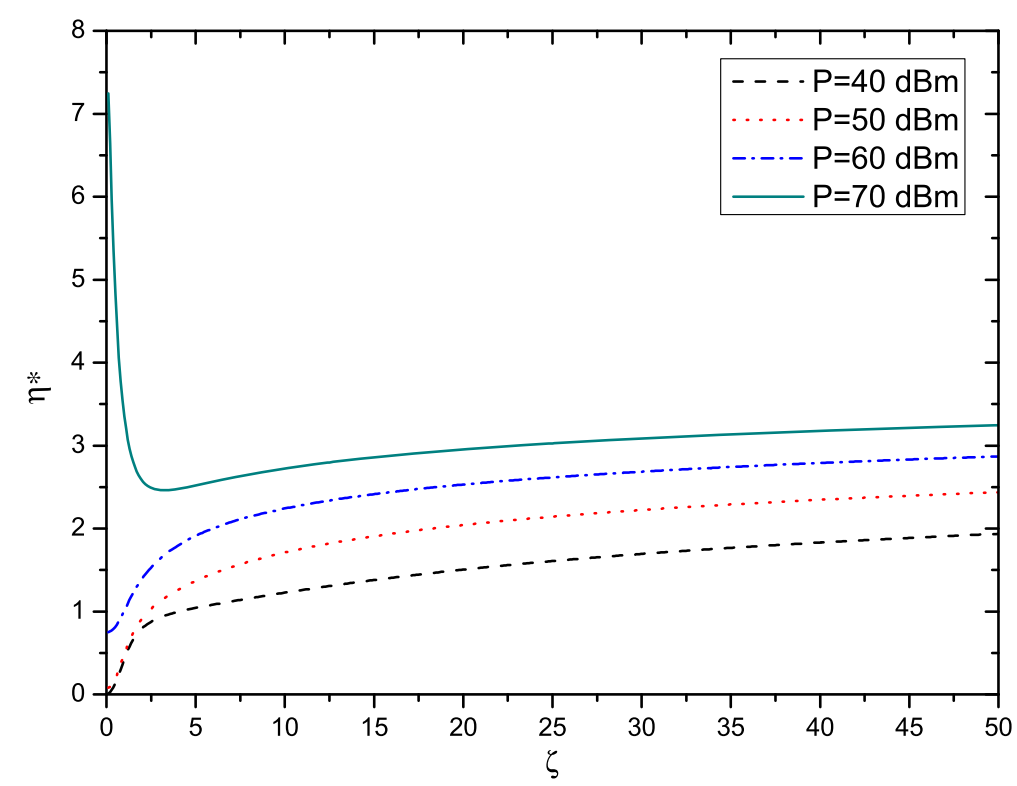

Fig. 4 The value of $\eta^{*}$ versus $\varsigma$ with different $P$ when $r=0.9, h=8.335 \times 10^{-7}$, and $\sigma=1$ 
minimize the BER will be investigated. By using the principle of convex optimization, the optimization problem is effectively solved. Finally, the implementation of the optimal tilting angle is discussed.

\subsection{Problem formulation}

The objective of the paper is to minimize the BER of the VLC system. According to the relative positions of LED and $\mathrm{PD}$, the tilting angle $\theta$ can not exceed $\pi / 2$, i.e., $\theta \in$ $[0, \pi / 2]$. Therefore, the optimization problem is given by

$$
\begin{array}{ll} 
& \min _{\theta} \text { BER } \\
\text { s.t. } & 0 \leq \theta \leq \pi / 2
\end{array}
$$

\subsection{Problem solving}

When $\varsigma^{2}>0$, the BER is given by (15); when $\varsigma^{2}=0$, the BER is given by (16). By analyzing (15) and (16), the following theorem can be derived.

Theorem 4 For all fixed $\varsigma^{2} \geq 0$, the BER for the VLC system is a monotonously decreasing function with $h$.

Proof See Appendix 4.

By using Theorem 4, problem (17) can be transformed to

$$
\begin{array}{ll} 
& \max _{\theta} h \\
\text { s.t. } & 0 \leq \theta \leq \pi / 2
\end{array}
$$

where the channel gain $h$ is given by (7). By analyzing (7), the following theorem can be obtained.

Theorem 5 The objection function $h$ in problem (18) is a concave function of $\theta$.

\section{Proof See Appendix 5.}

By using Theorem 5, the maximum value of $h$ is achieved by letting the first-order partial derivative of $h$ with $\theta$ to be zero, i.e.,

$$
\frac{\partial h}{\partial \theta}=\frac{(m+1) A(w-c)^{m}}{2 \pi d^{m+3}}\left[\sqrt{(u-a)^{2}+(v-b)^{2}} \cos \theta-|w-c| \sin \theta\right]=0
$$

Therefore, the optimal tilting angle $\theta^{*}$ is obtained as

$$
\theta^{*}=\arctan \left(\frac{\sqrt{(a-u)^{2}+(b-v)^{2}}}{|w-c|}\right)
$$

\subsection{Implementation of the tilting angle}

From (20), it can be found that the optimal tilting angle is a function of the positions of the LED and the PD. In this paper, the position of the LED (i.e., $[u, v, w]$ ) is fixed, but the position of the PD (i.e., $[a, b, c])$ is variable. Therefore, to realize the angle tilting of the receiver plane, the first step is to determine the position of the PD. With an arbitrary tilting angle larger than zero, the tilted receiver can obtain its position by using the three-dimensional positioning method proposed in our previous work [25]. After obtaining the position of the PD, the optimal tilting angle for the receiver can be calculated by using (20). Finally, according to the optimal tilting angle, the receiver can realize the angle tilting by employing an electrical machinery. The electrical machinery first makes the tilting angle to be zero and then increases the tilting angle until the optical tilting angle is achieved.

\section{Numerical results and discussion}

In this section, a practical VLC system in a room is considered as the test system. The derived theoretical expression of the BER will be confirmed by using the Monte-Carlo simulations in MATLAB. Note that the simulation results presented in this section are the average of $N=10^{6}$ independent trials. The detailed simulation process is provided in Algorithm 1. Moreover, the main simulation parameters are illustrated in Table 1.

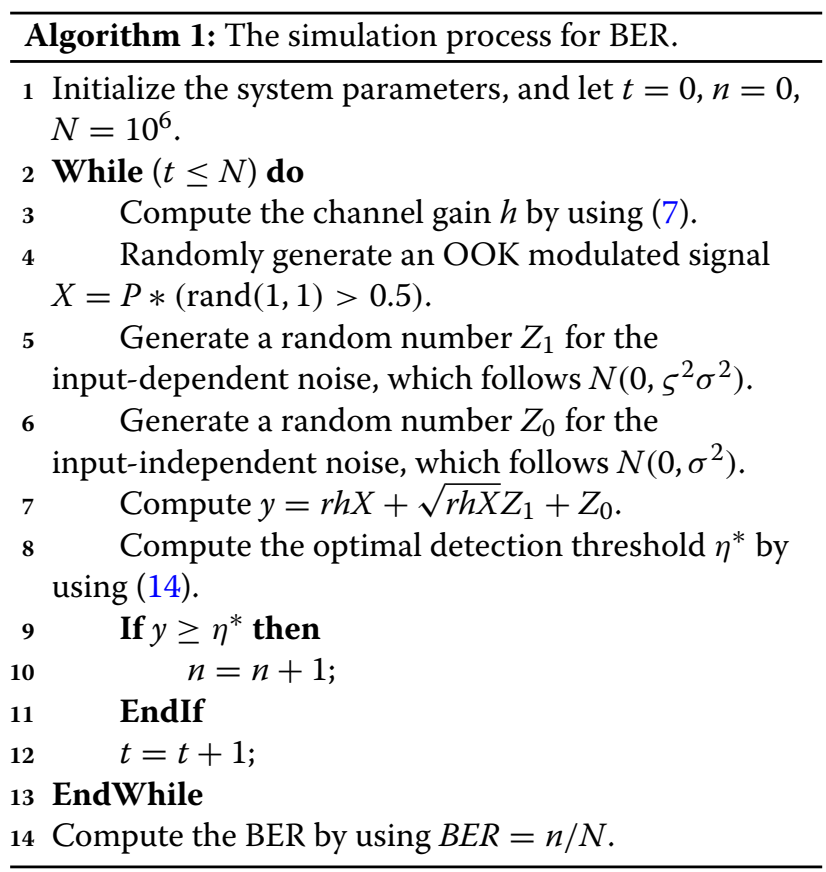

Table 1 Main simulation parameters

\begin{tabular}{lll}
\hline Descriptions & Symbols & Values \\
\hline Room size & $J \times K \times L$ & $8 \mathrm{~m} \times 8 \mathrm{~m} \times 3 \mathrm{~m}$ \\
Standard variance of noise & $\sigma$ & 1 \\
Physical area of PD & $A$ & $1 \mathrm{~cm}^{2}$ \\
Optoelectronic conversion factor of PD & $r$ & 0.9
\end{tabular}


Figure 5 shows the BER versus $\varsigma$ for different transmit optical powers $P$. In the simulation, $[u, v, w]=[2.5 \mathrm{~m}$, $2.5 \mathrm{~m}, 3 \mathrm{~m}]$ and $[a, b, c]=[2 \mathrm{~m}, 2 \mathrm{~m}, 1.5 \mathrm{~m}]$. The tilting angle of the PD is set to be zero. The order of the Lambertian emission $m=5$. It can be seen from the figure that, when $P$ is small, the BER performance improves with the increase of $\varsigma$. However, when $P$ is large, the value of the BER increases as $\zeta$. This indicates that the BER performance is strongly effected by the value of $\zeta$. Moreover, when $\varsigma$ tends to a large value, the value of the BER trends to a stable value (i.e., 0.25). From Fig. 5, for a fixed $\varsigma$, the BER performance improves with the increase of $P$. As is known, large optical power will generate a high SNR at receiver, and thus it will result in good BER performance. Furthermore, the theoretical results match exactly with the simulation results. This indicates that the derived BER expression can be used to evaluate the system performance without time-intensive simulations.

Figure 6 shows the BER versus the channel gain $h$ with different $\zeta$. In this simulation, the transmit optical power of the LED is $P=50 \mathrm{dBm}$. As can be observed, with the increase of $h$, the BER performance improves accordingly, which coincides with Theorem 4. Moreover, for small value of $h$ as shown in Fig. 6a, the BER performance improves with the increase of $\varsigma$. However, for large value of $h$ as shown in Fig. 6b', the BER performance degrades with the increase of the $\varsigma$. This conclusion is similar to that in Fig. 5. Once again, it should be noted that the theoretical results match well to the simulation results.
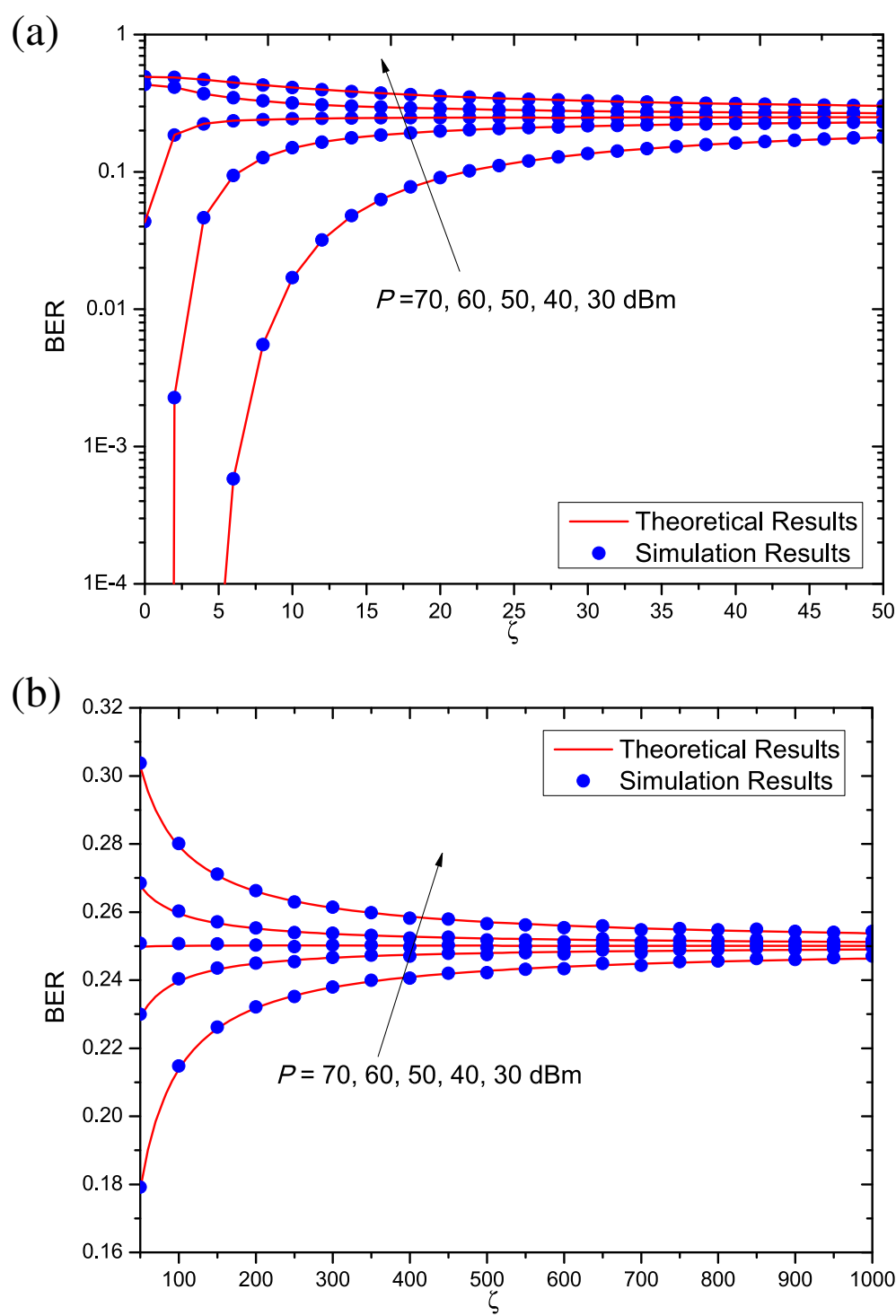

Fig. 5 BER versus $\varsigma$ with different transmit optical powers. a $\varsigma \in(0,50)$, b $\varsigma \in(50,1000)$ 

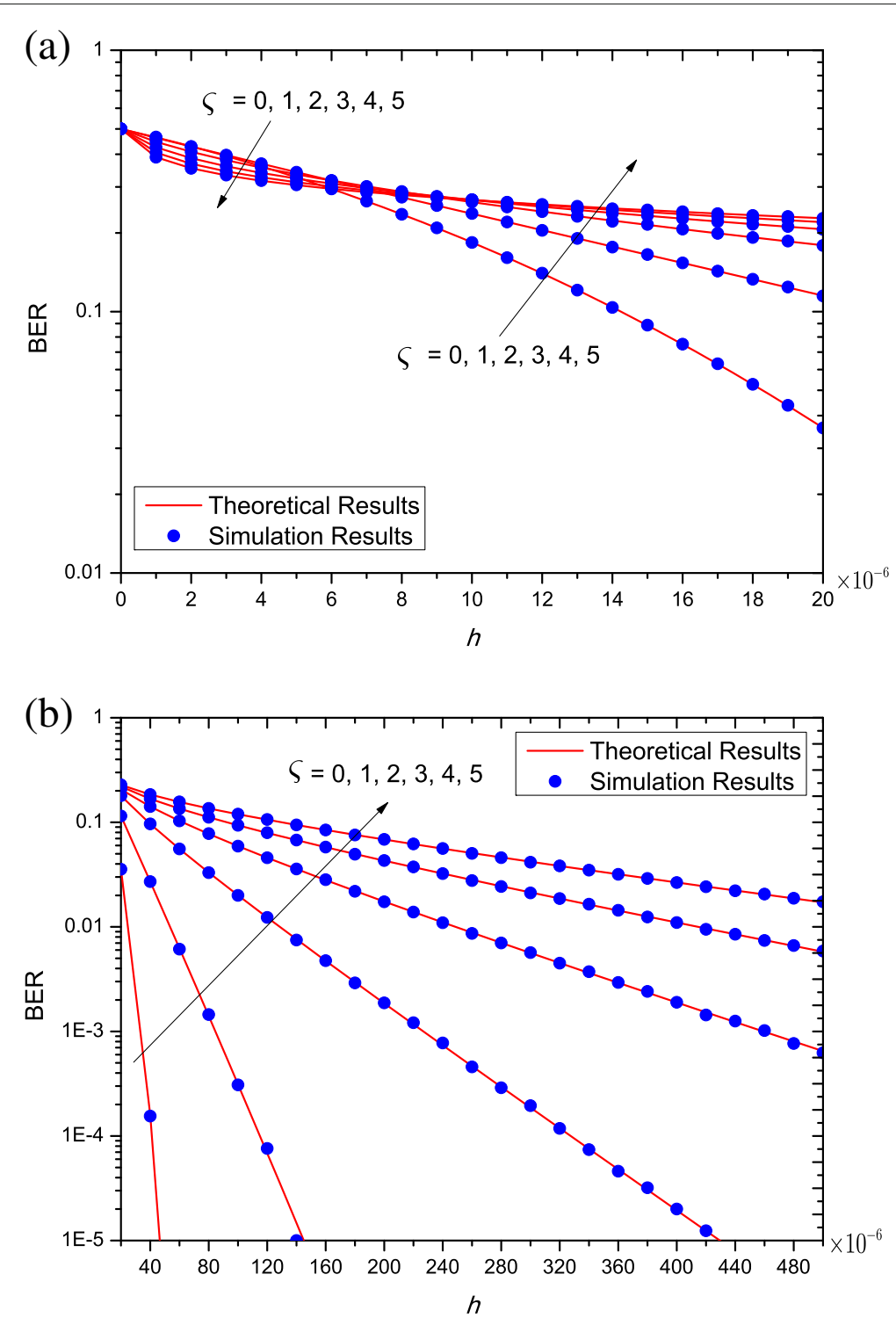

Fig. 6 BER versus channel gain $h$ with different $\varsigma$ when $P=50 \mathrm{dBm}$. a BER with small $h$, b BER with large $h$

Figure 7 shows the channel gain $h$ versus the semi-angle at half power of the LED $\Phi_{1 / 2}$ before and after tilting the receiver plane. In the simulation, the average optical power $P$ is set to be $50 \mathrm{dBm}$, and the coordinates of LED and PD are set to be $[2.5 \mathrm{~m}, 2.5 \mathrm{~m}, 3 \mathrm{~m}]$ and $[0 \mathrm{~m}$, $0 \mathrm{~m}, 0 \mathrm{~m}$ ], respectively. It can be observed that, as the increase of $\Phi_{1 / 2}$, both the two curves increase first and then decrease a little. When $\Phi_{1 / 2}=55^{\circ}$, the channel gains of the two curves achieve the maximum values. In addition, by tilting the receiver plane, the channel gain performance improves dramatically. This means that it is necessary to tilt the receiver plane properly to enhance system performance.

Figure 8 plots the distributions of the BER before and after tilting the receiver plane in the room. It can be observed that the worst BER achieves when the PD is located in the corner, while the best BER obtains when the PD is located in the center of the floor. This is $n$ because large transmission distance will result in the system performance degradation. Moreover, the BER performance improves in the whole room after tilting the receiver plane. Specifically, when the PD is located in the corner, the value of the BER reduces from 0.3487 to 0.2270 after tilting the receiver plane. This indicates that it is necessary to tilt the receiver plane properly in VLC system.

Figure 9 shows the distributions of tilting angles in the whole receiver plane after tilting the receiver plane. In this simulation, the LED is fixed on the center of the ceiling, while the PD can move to any place of the receiver plane. In Fig. 9, when the PD is located in the center of 


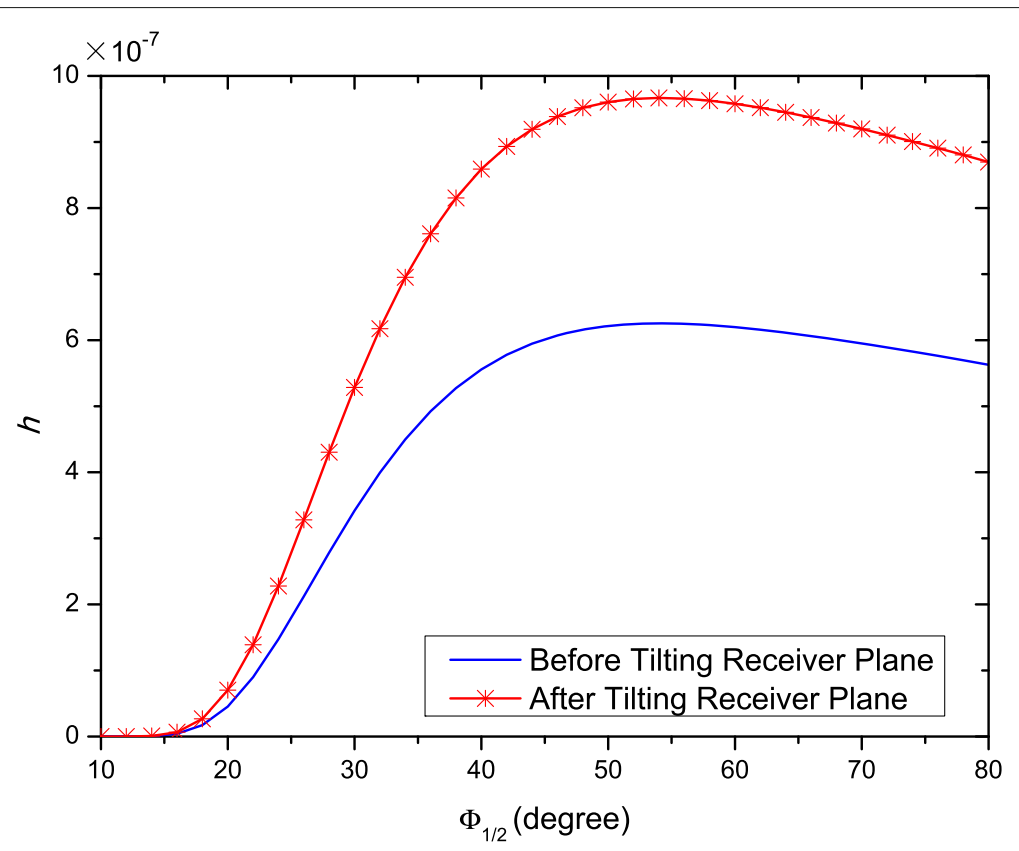

Fig. 7 Channel gain $h$ versus the semi-angle at half power of the LED $\Phi_{1 / 2}$

the receiver plane, the distance between the transceiver achieves the minimum value, and the tilting angle of the $\mathrm{PD}$ in this case is zero. When the PD moves from the center of the receiver plane to other place, the tilting angle of the PD increases gradually. When the PD is located in the corner of the receiver plane, the tilting angle of the PD gets its maximum value, i.e., $1.8032 \mathrm{rad}$. Therefore, the tilting angle provided in this figure can provide some insights for practical system design.

\section{Conclusions}

This paper focuses on an VLC system with inputdependent noise. The main conclusions of this paper are listed as follows:

- For the VLC system with input-dependent noise, the optimal detection threshold is obtained. Also, the theoretical expression of the BER is derived, which is quite accurate to evaluate the system performance.

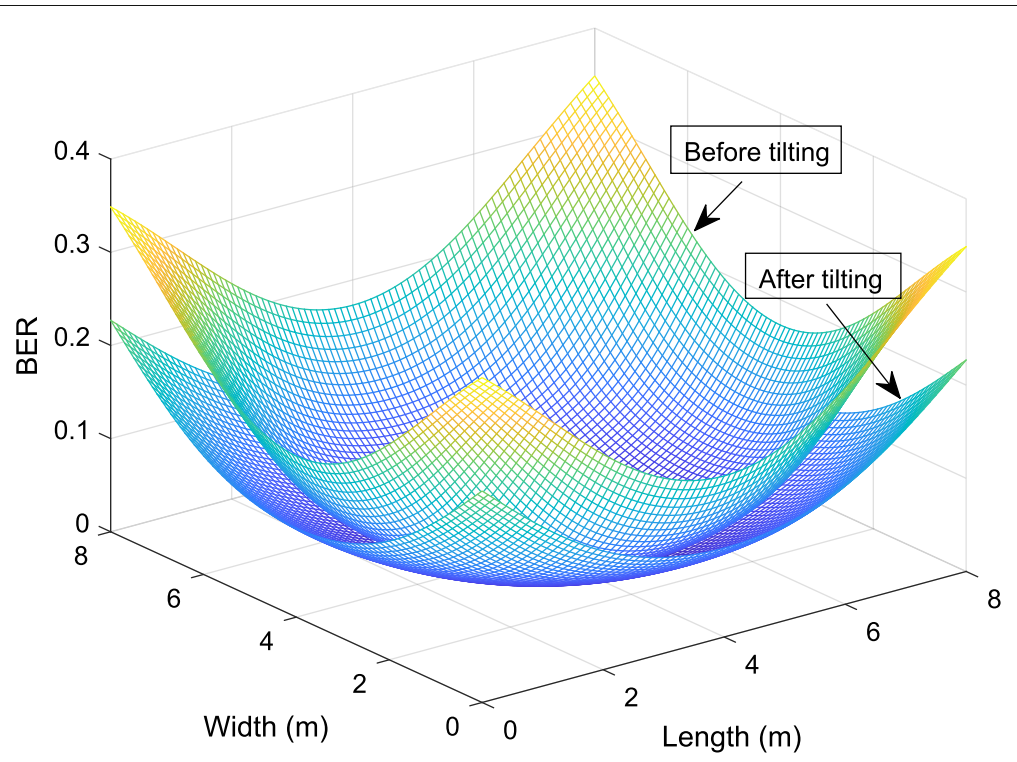

Fig. 8 BER distributions before and after tilting the receiver plane in the whole room 


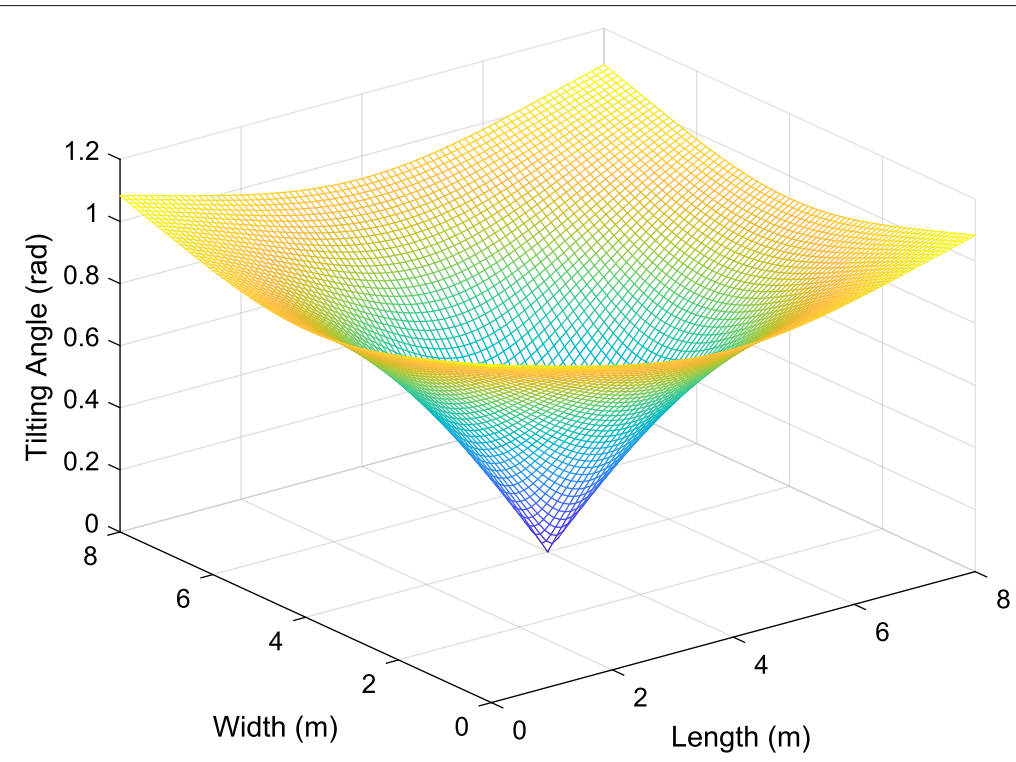

Fig. 9 Tilting angle distributions after tilting the receiver plane in the room

- The system performance is strongly affected by the input-dependent noise. For small $h$ or $P$, the BER decreases with the increase of $\zeta$. However, for large $h$ or $P$, the trend of the BER curve changes. Moreover, the larger the value of the channel gain is, the better the BER performance becomes.

- When the transceiver distance is large, the BER performance can be dramatically enhanced by tilting the receiver plane. In practice, the suggested tilting angle of the receiver place is shown in Fig. 9.

\section{Appendix 1: Proof of Theorem 1}

To obtain the optimum detection threshold, taking the first-order partial derivative of $B E R_{\varsigma^{2}>0}$ with respect to $\eta$ and letting it to be zero, we have

$$
\frac{\partial B E R_{\zeta^{2}>0}}{\partial \eta}=\frac{1}{2 \sqrt{2 \pi} \sigma}\left[\frac{e^{-\frac{(\chi-\eta)^{2}}{2\left(1+\chi \varsigma^{2}\right) \sigma^{2}}}}{\sqrt{1+\chi \varsigma^{2}}}-e^{-\frac{\eta^{2}}{2 \sigma^{2}}}\right]=0
$$

i.e.,

$$
\begin{aligned}
\frac{\chi \varsigma^{2}}{1+\chi \varsigma^{2}} \eta^{2} & +\frac{2 \chi}{1+\chi \varsigma^{2}} \eta-\frac{\chi^{2}}{1+\chi \varsigma^{2}} \\
& -\sigma^{2} \ln \left(1+\chi \varsigma^{2}\right)=0
\end{aligned}
$$

By solving the quadratic Eq. (22), we have

$$
\eta=\frac{-1 \pm \sqrt{1+\chi \varsigma^{2}+\frac{\varsigma^{2} \sigma^{2}\left(1+\chi \varsigma^{2}\right) \ln \left(1+\chi \varsigma^{2}\right)}{\chi}}}{\varsigma^{2}}
$$

According to the curve of BER in Fig. 3, the optimum detection threshold (14) is obtained.

\section{Appendix 2: Proof of Theorem 2}

Case (a): When $\chi$ is very small, we have

$$
\eta^{*} \approx \frac{\sqrt{1+\varsigma^{4} \sigma^{2}}-1}{\varsigma^{2}}
$$

Taking the first-order derivative of $\eta^{*}$ with respect to $\zeta$, we have

$$
\frac{\mathrm{d}}{\mathrm{d} \varsigma}\left(\frac{\sqrt{1+\varsigma^{4} \sigma^{2}}-1}{\varsigma^{2}}\right)=\frac{2\left(\sqrt{1+\varsigma^{4} \sigma^{2}}-1\right)}{\varsigma^{3} \sqrt{1+\varsigma^{4} \sigma^{2}}}>0
$$

This indicates that when $\chi$ is small, the value of $\eta^{*}$ rises with the increase of $\zeta$.

Case (b): When $\chi$ is very large, we have

$$
\eta^{*} \approx \frac{\sqrt{\chi+\varsigma^{2} \sigma^{2} \ln \left(\chi \varsigma^{2}\right)}}{\varsigma}
$$

Taking the first-order derivative of $\eta^{*}$ with respect to $\zeta$, we have

$$
\frac{\mathrm{d}}{\mathrm{d} \varsigma} \frac{\sqrt{\chi+\varsigma^{2} \sigma^{2} \ln \left(\chi \varsigma^{2}\right)}}{\varsigma}=\frac{\varsigma^{2} \sigma^{2}-\chi}{\varsigma^{2} \sqrt{\chi+\varsigma^{2} \sigma^{2} \ln \left(\chi \varsigma^{2}\right)}}<0
$$

This indicates that when $\chi$ is large, the value of $\eta^{*}$ decreases with the increase of $\varsigma$. 


\section{Appendix 3: Proof of Theorem 3}

Case (a): When $\varsigma^{2} \rightarrow 0$, using the L'Hospital's rule, we have

$$
\begin{aligned}
\lim _{\varsigma \rightarrow 0} \frac{\sqrt{1+\chi \varsigma^{2}}-\sqrt{1+\frac{\varsigma^{2} \sigma^{2} \ln \left(1+\chi \varsigma^{2}\right)}{\chi}}}{\varsigma^{2} \sigma \quad} & =\lim _{\varsigma \rightarrow 0}\left[\frac{\chi}{2 \sigma \sqrt{1+\chi \varsigma^{2}}}-\frac{\sigma \ln \left(1+\chi \varsigma^{2}\right)+\frac{\chi \varsigma^{2} \sigma}{1+\chi \varsigma^{2}}}{2 \chi \sqrt{1+\frac{\varsigma^{2} \sigma^{2} \ln \left(1+\chi \varsigma^{2}\right)}{\chi}}}\right] \\
& =\frac{\chi}{2 \sigma}
\end{aligned}
$$

and

$$
\begin{aligned}
& \lim _{\varsigma \rightarrow 0} \frac{\sqrt{1+\chi \varsigma^{2}+\frac{\varsigma^{2} \sigma^{2}\left(1+\chi \varsigma^{2}\right) \ln \left(1+\chi \varsigma^{2}\right)}{\chi}}-1}{\varsigma^{2} \sigma} \\
& =\lim _{\varsigma \rightarrow 0} \frac{\frac{\chi}{2}+\frac{\left(2 \varsigma \sigma^{2}+4 \chi \varsigma^{3} \sigma^{2}\right) \ln \left(1+\chi \varsigma^{2}\right)+\left(\varsigma^{2} \sigma^{2}+\chi \varsigma^{4} \sigma^{2}\right) \frac{\chi}{2\left(1+\chi \varsigma^{2}\right)}}{\chi}}{\sigma \sqrt{1+\chi \varsigma^{2}+\frac{\varsigma^{2} \sigma^{2}\left(1+\chi \varsigma^{2}\right) \ln \left(1+\chi \varsigma^{2}\right)}{\chi}}} \\
& =\frac{\chi}{2 \sigma}
\end{aligned}
$$

Submitting (28) and (29) into (15), the asymptotic BER is given by

$$
\lim _{\varsigma \rightarrow 0} B E R_{\varsigma^{2}>0}=\mathcal{Q}\left(\frac{\chi}{2 \sigma}\right)
$$

Case (b): When $\varsigma^{2}=0$, the VLC system only includes the input-independent noise. Under this circumstance, (8) reduces to $Y=r h X+Z_{0}$. According to Theorem 1, the optimum detection threshold in this case can be easily derived as $\eta^{*}=\chi / 2$. Moreover, the conditional PDF $f_{Y \mid X}(y \mid x)$ becomes

$$
f_{Y \mid X}(y \mid x)=\frac{1}{\sqrt{2 \pi} \sigma} \exp \left[-\frac{(y-r h x)^{2}}{2 \sigma^{2}}\right]
$$

Therefore, the BER becomes

$$
\begin{aligned}
\operatorname{BER}_{\varsigma^{2}=0} & =\frac{1}{2}\left[\int_{-\infty}^{\chi / 2} \frac{1}{\sqrt{2 \pi} \sigma} e^{-\frac{(y-\chi)^{2}}{2 \sigma^{2}}} \mathrm{~d} y+\int_{\chi / 2}^{\infty} \frac{1}{\sqrt{2 \pi} \sigma} e^{-\frac{y^{2}}{2 \sigma^{2}}} \mathrm{~d} y\right] \\
& =\mathcal{Q}\left(\frac{\chi}{2 \sigma}\right)
\end{aligned}
$$

According to (30) and (32), Theorem 3 holds.

\section{Appendix 4: Proof of Theorem 4}

Define $I_{1}$ and $I_{2}$ as

$$
\left\{\begin{array}{l}
I_{1}=\frac{1}{\varsigma^{2} \sigma}(\underbrace{\sqrt{1+\chi \varsigma^{2}}}_{I_{3}}-\underbrace{\sqrt{1+\frac{\varsigma^{2} \sigma^{2} \ln \left(1+\chi \varsigma^{2}\right)}{\chi}}}_{I_{4}}) \\
I_{2}=\frac{\sqrt{1+\chi \varsigma^{2}+\frac{\varsigma^{2} \sigma^{2}\left(1+\chi \varsigma^{2}\right) \ln \left(1+\chi \varsigma^{2}\right)}{x}}-1}{\varsigma^{2} \sigma}
\end{array}\right.
$$

In $I_{1}$, taking the first-order partial derivative of $I_{4}$ with $h$, we can get

$$
\frac{\partial I_{4}}{\partial h}=\frac{1}{2 \sqrt{1+\frac{\varsigma^{2} \sigma^{2} \ln \left(1+\chi \varsigma^{2}\right)}{\chi}}} \frac{\varsigma^{2} \sigma^{2}}{\chi h} \underbrace{\left[\frac{\chi \varsigma^{2}}{1+\chi \varsigma^{2}}-\ln \left(1+\chi \varsigma^{2}\right)\right]}_{I_{5}}
$$

Then, taking the first-order partial derivative of $I_{5}$ with $h$, we can get

$$
\begin{aligned}
\frac{\partial I_{5}}{\partial h} & =\frac{2 r P \varsigma^{2}\left(1+\chi \varsigma^{2}\right)-2 r P \varsigma^{2} \chi \varsigma^{2}}{\left(1+\chi \varsigma^{2}\right)^{2}}-\frac{2 r P \varsigma^{2}}{1+\chi \varsigma^{2}} \\
& =\frac{-2 r P \chi \varsigma^{4}}{\left(1+\chi \varsigma^{2}\right)^{2}}<0
\end{aligned}
$$

which indicates that $I_{5}$ is a monotonously decreasing function of $h$. When $h \rightarrow 0, I_{5} \rightarrow 0$. Since $h>0, I_{5}<0$ always holds. Moreover, according to (34), we have $\partial I_{4} / \partial h \leq$ 0 , which means that $I_{4}$ is a monotonously decreasing function of $h$. Moreover, $I_{3}$ is a monotonously increasing function of $h$. Given the above, $I_{1}$ is a monotonously increasing function of $h$.

Furthermore, taking the first-order partial derivative of $I_{2}$ with $h$, we have

$$
\frac{\partial I_{2}}{\partial h}=\frac{1}{\varsigma^{2} \sigma} \frac{2 r P \varsigma^{2}+\frac{\varsigma^{2} \sigma^{2}}{\chi h}\left[\chi \varsigma^{2}-\ln \left(1+\chi \varsigma^{2}\right)\right]}{2 \sqrt{1+\chi \varsigma^{2}+\frac{\varsigma^{2} \sigma^{2}\left(1+\chi \varsigma^{2}\right) \ln \left(1+\chi \varsigma^{2}\right)}{\chi}}}>0(36)
$$

where the inequality follows because $x>\ln (1+x)$ always holds for all $x>0$. Therefore, $I_{2}$ is also a monotonously increasing function of $h$. Moreover, $Q(\cdot)$ is a monotonously decreasing function. Therefore, $\mathrm{BER}_{\varsigma^{2}>0}$ is a monotonously decreasing function of $h$, i.e.,

$$
\frac{\partial \mathrm{BER}_{\varsigma^{2}}>0}{\partial h}<0
$$

Similarly, when $\varsigma^{2}=0$, taking the first-order partial derivative of $\mathrm{BER}_{\varsigma^{2}=0}$ with respect to $h$ in (16), we have

$$
\frac{\partial \mathrm{BER}_{\varsigma^{2}=0}}{\partial h}=-\frac{e^{-\frac{\chi^{2}}{4 \sigma^{2}}}}{\sqrt{2 \pi}} \frac{r P}{\sigma}<0
$$

According to (37) and (38), Theorem 4 can be obtained.

\section{Appendix 5: Proof of Theorem 5}

By taking the second-order partial derivative of $h$ with $\theta$, we get

$$
\begin{aligned}
\frac{\partial^{2} h}{\partial \theta^{2}}= & -\frac{(m+1) A(w-c)^{m}}{2 \pi d^{m+3}}[(u-a) \cos \varphi \sin \theta \\
& +(v-b) \sin \varphi \sin \theta+(w c) \cos \theta]=-h
\end{aligned}
$$

In VLC, $h$ is a non-negative number, and thus $\partial^{2} h / \partial \theta^{2}<0$. Therefore, Theorem 5 always holds. 


\section{Abbreviations}

ACO: Asymmetrically clipped optical; BER: Bit error rate; CSK: Color shift keying; DCO: Direct current optical; GSSK: Generalized space shift keying; LEDs: Light-emitting diodes; OFDM: Orthogonal frequency division multiplexing; OOK: On-off keying; PD: Photodiodes; SNR: Signal-to-noise ratio; VLC: Visible light communication

\section{Acknowledgements}

The research presented in this paper was supported by Huaiyin Institute of Technology, China.

\section{Funding}

This paper is supported by National Natural Science Foundation of China (61701254), Natural Science Foundation of Jiangsu Province (BK20170901), Key International Cooperation Research Project (61720106003), the open fund for Jiangsu key laboratory of traffic and transportation security, Huaiyin Institute of Technology (TTS2017-03), the Open Research Fund of the Key Laboratory of Broadband Wireless Communication and Sensor Network Technology, Nanjing University of Posts and Telecommunications, the Ministry of Education (JZNY201706), NUPTSF (NY216009), the Open Research Fund of the Key Laboratory of Intelligent Computing \& Signal Processing, Anhui University, and the Open Research Subject of the Key Laboratory (Research Base) of Signal and Information Processing, Xihua University (szjj2017-047).

\section{Availability of data and materials}

The authors declare that all the data and materials in this manuscript are available.

\section{Authors' contributions}

$\mathrm{SHL}$ is the main contributor of the paper. She proposes the main idea, derives the expression of $B E R$, and solves the optimization problem. $C L$ helps to finish the simulation results. XB and JYW help to polish the content of the paper. All authors read and approve the final manuscript.

\section{Authors' information}

Sheng-Hong Lin is working toward her PhD degree at Nanjing University of Posts and Telecommunications, Nanjing, China. Her research interest is visible light communications.

Cheng Liu is currently studying for the M.S. degree from the National Mobile Communications Research Laboratory, Southeast University, Nanjing, China. His research interest is physical-layer security in visible light communications. Xu Bao is currently a professor at Jiangsu Key Laboratory of Traffic and Transportation Security, Huaiyin Institute of Technology, Huai'an, China. His research interest is visible light communications.

Jin-Yuan Wang is currently a lecturer at College of Telecommunications and Information Engineering, Nanjing University of Posts and Telecommunications, Nanjing, China. His current research interest is visible light communications.

\section{Competing interests}

The authors declare that they have no competing interests.

\section{Publisher's Note}

Springer Nature remains neutral with regard to jurisdictional claims in published maps and institutional affiliations.

\section{Author details \\ 1 Jiangsu Key Laboratory of Traffic and Transportation Security, Huaiyin Institute of Technology, 223003 Huaian, China. ${ }^{2}$ Key Lab of Broadband Wireless Communication and Sensor Network Technology, Nanjing University of Posts and Telecommunications, 210003 Nanjing, China. ${ }^{3}$ National Mobile Communications Research Laboratory, Southeast University, 211111 Nanjing, China.}

Received: 22 May 2018 Accepted: 5 September 2018 Published online: 21 September 2018

\section{References}

1. P. H. Rathak, X. Feng, P. Hu, P. Mohapatra, Visible light communication, networking and sensing: a survey, potential and challenges. IEEE Commun. Surv. \& Tutorials. 17(4), 2047-2076 (2015)
2. H. Hass, L. Yin, Y. Wang, C. Chen, What is LiFi? IEEE/OSA J. Lightwave Technol. 34(6), 1533-1544 (2016)

3. C. P. Li, Y.ing. Yi, K. S. Lee, Performance analysis of visible light communication using the STBC-OFDM technique for intelligent transportation systems. Int. J. Electron. 101(8), 1117-1133 (2014)

4. R. M. Mare, C. Luiz Marte, C. E. Cugnasca, Visible light communication applied to intelligent transport systems: an overview. IEEE Lat. Am. Trans. 14(7), 3199-3207 (2016)

5. T. Komine, M. Nakagawa, Fundamental analysis for visible-light communication system using LED lights. IEEE Trans. Consum. Electron. 50(1), 100-107 (2004)

6. J. B. Wang, Q. S. Hu, J. Wang, M. Chen, J. Y. Wang, Tight bounds on channel capacity for dimmable visible light communications. IEEE/OSA J. Lightwave Technol. 31(23), 3771-3779 (2013)

7. R. Jiang, Z. Wang, Q. Wang, L. Dai, A tight upper bound on channel capacity for visible light communications. IEEE Commun. Lett. 20(1), 97-100 (2016)

8. K. I.1. Ahn, J. K. Kwon, Capacity analysis of M-PAM inverse source coding in visible light communications. IEEE/OSA J. Lightwave Technol. 30(10), 1399-1404 (2012)

9. X. Li, J. Vucic, V. Jungnickel, J. Armstrong, On the capacity of intensity-modulated direct-detection systems and the information rate of ACO-OFDM for indoor optical wireless applications. IEEE Trans. Commun. 60(3), 799-809 (2012)

10. C. P. Li, Y.ing. Yi, K. S. Lee, Pilot self-coding applied in optical OFDM systems. Int. J. Electron. 102(4), 548-562 (2015)

11. L. Jia, J. Y. Wang, W. Zhang, M. Chen, J. B. Wang, Symbol error rate analysis for colour-shift keying modulation in visible light communication system with RGB light-emitting diodes. IET Optoelectron. 9(5), 199-206 (2015)

12. W. O. Popoola, E. Poves, H. Haas, Error performance of generalised space shift keying for indoor visible light communications. IEEE Trans. Commun. 61(5), 1968-1976 (2013)

13. Y. Yi, C. P. Li, K. S. Lee, Optimum spread code applied in indoor visible light data transmission for optical multipath dispersion reduction. IETE Techn. Rev. 30(3), 233-239 (2013)

14. Y. Yi, C. P. Li, K. S. Lee, Adaptive MMSE equalizer for optical multipath dispersion in indoor visible light communication. IETE J. Res. 58(5), 347-355 (2012)

15. J. Katz, Detectors for optical communications: A review. Adv. Mater. 5(1), 21-38 (1983)

16. J. Y. Wang, J. B. Wang, M. Chen, J. Wang, in IEEE Global Communications Conference. Capacity bounds for dimmable visible light communications using PIN photodiodes with input-dependent Gaussian noise (IEEE, Austin, 2014), pp. 1-6

17. J. Y. Wang, Z. Yang, Y. Wang, M. Chen, On the performance of spatial modulation based optical wireless communications. IEEE Photon. Technol. Lett. 28(19), 2094-2097 (2016)

18. M. F. Keskin, S. Gezici, Comparative theoretical analysis of distance estimation in visible light positioning systems. IEEE/OSA J. Lightwave Technol. 34(3), 854-865 (2016)

19. K. Xu, H. Yu, Y. J. Zhu, Channel-adpted spatial modulation for massive MIMO visible light communications. IEEE Photon. Technol. Lett. 28(23), 2693-2696 (2016)

20. W. Xu, J. Wang, H. Shen, H. Zhang, X. You, Indoor positioning for multiphotodiode device using visible-light communications. IEEE Photon. J. 8(1), 1-11 (2016)

21. Z. Wang, C. Yu, W. D. Zhong, J. Chen, Performance improvement by tilting receiver plane in M-QAM OFDM visible light communications. Opt. Exp. 19(14), 13418-13427 (2011)

22. J. Y. Wang, J. Dai, R. Guan, L. Jia, Y. Wang, M. Chen, Channel capacity and receiver deployment optimization for multi-input multi-output visible light communications. Opt. Exp. 24(12), 1-15 (2016)

23. A. Lapidoth, S. M. Moser, M. A. Wigger, On the capacity of free-space optical intensity channels. IEEE Trans. Inf. Theory. 55(10), 4449-4461 (2009)

24. S. M. Navidpour, M. Uysal, M. Kavehrad, BER performance of free-space optical transmission with spatial diversity. IEEE Trans. Wirel. Commun. 6(8), 2813-2819 (2007)

25. Q. L. Li, J. Y. Wang, T. Huang, Y. Wang, Three-dimensional indoor visible light positioning system with a single transmitter and a single tilted receiver. Opt Eng. 55(10), 106103-1-7 (2016) 